\title{
Synthese und Struktur von Phenyltetra(acetato)stiboran
}

\author{
Synthesis and Structure of Phenyltetra(acetato)stiborane \\ Markus Wieber*, Isa Fetzer-Kremling, Hildegard Reith und Christian Burschka \\ Institut für Anorganische Chemie der Universität Würzburg, Am Hubland, D-8700 Würzburg \\ Z. Naturforsch. 42b, 815-817 (1987); eingegangen am 2. März/6. Mai 1987 \\ Phenyltetra(acetato)stiborane, Synthesis, X-Ray Crystal Structure \\ Phenyltetra(acetato)stiborane is obtained by reacting phenyldichlorostibane successively with \\ sulfuryl chloride and silver acetate at low temperatures $\left(-70{ }^{\circ} \mathrm{C}\right)$. An $\mathrm{X}$-ray analysis of \\ $\mathrm{C}_{6} \mathrm{H}_{5} \mathrm{Sb}\left[\mathrm{OC}(\mathrm{O}) \mathrm{CH}_{3}\right]_{4}$ shows, that the central antimony atom is hexacoordinated by chelation of \\ one nearly symmetrically bonded acetate group, a phenyl group, and three singly-bonded acetate \\ ligands.
}

\section{Einleitung}

Tetraorganostiboniumcarboxylate [1] und Triorganodicarboxylatostiborane [2] sind bekannte Verbindungsklassen. Von Tetramethylstibonium-Hydrogendicarboxilaten [3], Triphenyldi(acetato)stiboran [4] und Triphenyldi(benzoato)stiboran [5] existieren Kristallstrukturanalysen. Von entsprechenden Diorganotri(carboxylato)stiboranen sind nur wenige Alkylsubstituierte Verbindungen bekannt. Spektroskopische Untersuchungen lassen auf eine oktaedrische Struktur mit chelatisierenden Azetato-Liganden schließen [6]. Versuche zur Darstellung der entsprechenden Phenylverbindung führten zu einem teilweise hydrolysierten Produkt, dessen Kristallstruktur bestimmt wurde [7].

Unbekannt sind bisher Monoorganotetra(carboxylato)stiborane. Über die Synthese und die Kristallstruktur eines ersten Beispiels dieser Verbindungsklasse wird hier berichtet.

\section{Synthese und Eigenschaften von Phenyltetra(acetato)stiboran}

Phenyltetrachlorostiboran wurde intermediär durch Einwirken von frisch destilliertem Sulfurylchlorid auf Phenyldichlorstiban in Methylenchlorid bei $-70{ }^{\circ} \mathrm{C}$ gebildet und nachfolgend spatelweise mit Silberacetat versetzt.

$$
\begin{aligned}
& \mathrm{C}_{6} \mathrm{H}_{5} \mathrm{SbCl}_{2}+\mathrm{SO}_{2} \mathrm{Cl}_{2}+4 \mathrm{AgOOCCH} \\
& \mathrm{C}_{6} \mathrm{H}_{5} \mathrm{Sb}\left(\mathrm{OOCCH}_{3}\right)_{4}+4 \mathrm{AgCl}+\mathrm{SO}_{2}
\end{aligned}
$$

Phenyltetra(acetato)stiboran fällt in Form eines farblosen Pulvers an. Zur Reinigung löst man in

\footnotetext{
* Sonderdruckanforderungen an Prof. Dr. Markus Wieber.

Verlag der Zeitschrift für Naturforschung, D-7400 Tübingen 0932-0776/87/0700-0815/\$01.00/0
}

Methylenchlorid, gibt Pentan zu und erhält farblose Kristalle, die sich in mäßig polaren Lösungsmitteln, wie $\mathrm{CH}_{2} \mathrm{Cl}_{2}, \mathrm{CHCl}_{3}$ und in Benzol sowie in $\mathrm{CCl}_{4}$ gut lösen. Phenyltetra(acetato)stiboran besitzt einen Schmelzpunkt von $117^{\circ} \mathrm{C}$ und ist äußerst hydrolyseempfindlich.

Das ${ }^{1} \mathrm{H}$-NMR-Spektrum wurde in Methylenchlorid bei $30{ }^{\circ} \mathrm{C}$ und $-60{ }^{\circ} \mathrm{C}$ aufgenommen und zeigt die erwarteten Signale für die Phenylprotonen im Bereich von 7,38-7,75 ppm sowie 7,83-8,22 ppm und ein Singulett für die Acetatprotonen bei 2,00 ppm.

Auf Grund der leichten Zersetzlichkeit war eine Molekulargewichtsbestimmung nicht durchführbar. Das bei $80^{\circ} \mathrm{C}$ vermessene Massenspektrum wies nur Zersetzungsprodukte auf.

\section{Strukturuntersuchung und Diskussion}

Es ist von mehreren Beispielen bekannt, daß Carboxylatreste in Verbindungen der schweren $\mathrm{Vb}$ Elemente sowohl inter- als auch intramolekular als zweizähnige Liganden fungieren können [8-10]. Für strukturelle Untersuchungen ist deshalb im IR-Spektrum insbesondere die Lage der $\mathrm{C}=\mathrm{O}$-Schwingungsbande von Interesse.

Im Carbonylbereich wird in $\mathrm{CCl}_{4}$ bei $1717 \mathrm{~cm}^{-1}$ eine starke Bande für die asymmetrische $\mathrm{C}=\mathrm{O}-\mathrm{Va}$ lenzschwingung gefunden, für die symmetrische eine schwächere bei $1365 \mathrm{~cm}^{-1}$; die C-O-Valenzschwingung liegt bei $1250 \mathrm{~cm}^{-1}$. Diese Werte sprechen für einen einzähnigen Acetatrest. Ob darüber hinaus chelatartige Bindungen bestehen, kann allein aus dem IR-Spektrum nicht geschlossen werden. Es wird zwar bei $1562 \mathrm{~cm}^{-1}$ eine Bande beobachtet, die durch bindungsäquivalente Carboxylatsauerstoffatome hervorgerufen werden könnte. Eine Zuordnung ist jedoch unsicher, da zwischen $1500 \mathrm{~cm}^{-1}$ und $1600 \mathrm{~cm}^{-1}$ 
auch C-C-Absorptionen der Phenylgruppe auftreten.

Aufschluß über das Koordinationsverhalten ergab eine Röntgenstrukturanalyse.

Die untersuchten, farblosen transparenten Kristalle überzogen sich auch unter Schutzgas langsam mit einer weiß-opaken bröseligen Schicht von Zersetzungsprodukten, die mechanisch entfernt werden konnte. Das erhaltene Strukturmodell zeigt Abb. 1 zusammen mit der Numerierung der Atome.

Tab. II enthält eine Auswahl der Abstände und Winkel, wie sie aus den in Tab. I aufgeführten Koordinaten resultieren. Die gewonnenen Daten zeigen deutlich verschiedene Koordination der Acetatgruppen: Während die Gruppen B, C und D stark asymmetrisch und annähernd einzähnig gebunden sind, koordiniert Gruppe A chelatartig zweizähnig und fast symmetrisch. Die Hauptkoordinationsebene im Molekül wird gebildet von den Atomen Sb, C3, O1D, O1A, O2A mit Bindungslängen zwischen 1,99 und 2,19 $\AA$ und Abweichungen der genannten Atome von dieser Ebene bis zu 0,06 $\AA$. Die beiden anderen Atome O1B und O1C der ersten Koordinationssphäre stehen mit Abständen von jeweils $1,99 \AA$ (von dieser Ebene) grob angenähert senkrecht darüber bzw. darunter, so daß sich die Koordination des Zentralatoms als verzerrt oktaedrisch beschreiben läßt. Allerdings wäre auch die Formulierung einer trigonalen bipyramidalen Anordnung denkbar, wenn man akzeptiert, daß die Acetat-

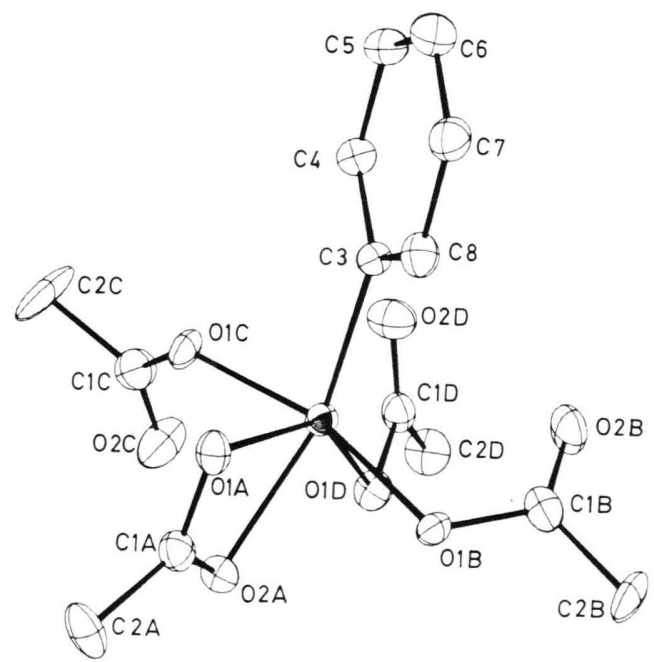

Abb. 1. ORTEP-PLOT der Molekülstruktur von Phenyltetra(acetato)stiboran $20 \%$ ). gruppe A eine equatoriale Koordinationsstelle symmetrisch besetzt.

Plausibel ist, daß über dem größten offenen Winkel dieser Koordination, nämlich $\mathrm{C} 3-\mathrm{Sb}-\mathrm{O} 1 \mathrm{D}$ $\left(129,3^{\circ}\right)$ das nächst entfernte O-Atom O2D (Abstand 2,7 $\AA$ ) zu finden ist, jedoch kann man es auf Grund des deutlich größeren Abstandes allenfalls

Tab. I. Atomkoordinaten und isotrope Temperaturfaktoren mit geschätzten Standardabweichungen in Einheiten der letzten Stelle in Klammern.

\begin{tabular}{lclll}
\hline Atom & $x$ & $y$ & $z$ & $\mathrm{~B}(\mathrm{eq})$ \\
\hline Sb & $0,18851(6)$ & $0,38900(3)$ & $0,27093(3)$ & $3,07(2)$ \\
O1 A & $0,0718(6)$ & $0,3131(3)$ & $0,1536(3)$ & $3,8(2)$ \\
O2A & $0,1793(6)$ & $0,4376(3)$ & $0,1256(3)$ & $3,8(2)$ \\
O1B & $0,3926(5)$ & $0,3331(3)$ & $0,2211(3)$ & $3,7(2)$ \\
O2B & $0,4943(6)$ & $0,3393(4)$ & $0,3710(4)$ & $5,3(3)$ \\
O1C & $-0,0324(5)$ & $0,4442(3)$ & $0,2771(3)$ & $4,2(3)$ \\
O2C & $0,0193(8)$ & $0,5855(4)$ & $0,2597(5)$ & $7,4(4)$ \\
O1D & $0,3220(6)$ & $0,4948(3)$ & $0,2971(3)$ & $4,1(3)$ \\
O2D & $0,2137(8)$ & $0,4800(4)$ & $0,4358(4)$ & $6,0(3)$ \\
C1A & $0,1026(9)$ & $0,3714(5)$ & $0,0921(5)$ & $3,9(4)$ \\
C2A & $0,0499(11)$ & $0,3641(5)$ & $-0,0120(6)$ & $6,0(5)$ \\
C1B & $0,5120(9)$ & $0,3207(5)$ & $0,2869(6)$ & $3,8(4)$ \\
C2B & $0,6682(9)$ & $0,2838(5)$ & $0,2504(6)$ & $5,4(5)$ \\
C1C & $-0,0737(11)$ & $0,5282(6)$ & $0,2738(6)$ & $5,3(5)$ \\
C2C & $-0,2558(11)$ & $0,5397(6)$ & $0,2898(10)$ & $9,8(7)$ \\
C1D & $0,3012(10)$ & $0,5221(5)$ & $0,3859(6)$ & $4,2(4)$ \\
C2D & $0,3870(10)$ & $0,6029(5)$ & $0,4157(6)$ & $5,6(4)$ \\
C3 & $0,1233(8)$ & $0,2906(5)$ & $0,3658(5)$ & $3,2(3)$ \\
C4 & $0,0292(9)$ & $0,3099(5)$ & $0,4449(6)$ & $4,7(4)$ \\
C5 & $-0,0206(10)$ & $0,2419(6)$ & $0,5037(6)$ & $5,4(5)$ \\
C6 & $0,0204(10)$ & $0,1560(6)$ & $0,4819(6)$ & $5,6(5)$ \\
C7 & $0,1110(9)$ & $0,1371(5)$ & $0,4016(6)$ & $4,6(4)$ \\
C8 & $0,1647(9)$ & $0,2059(5)$ & $0,3436(5)$ & $4,1(4)$ \\
\hline & & & &
\end{tabular}

Tab. II. Auswahl der interatomaren Abstände [^] und Winkel $\left[{ }^{\circ}\right]$ in der Kristallstruktur von Phenyltetra(acetato)stiboran.

\begin{tabular}{|c|c|c|c|}
\hline $\mathrm{Sb}-\mathrm{O} 1 \mathrm{~A}$ & $2,189(5)$ & $\mathrm{O} 1 \mathrm{~A}-\mathrm{Sb}-\mathrm{O} 2 \mathrm{~A}$ & $59,5(2)$ \\
\hline $\mathrm{Sb}-\mathrm{O} 2 \mathrm{~A}$ & $2,152(4)$ & $\mathrm{O} 1 \mathrm{~A}-\mathrm{Sb}-\mathrm{C} 3$ & $88,1(2)$ \\
\hline $\mathrm{Sb}-\mathrm{O} 1 \mathrm{~B}$ & $2,029(5)$ & $\mathrm{C} 3-\mathrm{Sb}-\mathrm{O} 1 \mathrm{D}$ & $129,3(2)$ \\
\hline $\mathrm{Sb} \cdots \mathrm{O} 2 \mathrm{~B}$ & $2,904(5)$ & $\mathrm{O} 1 \mathrm{D}-\mathrm{Sb}-\mathrm{O} 2 \mathrm{~A}$ & $83,1(2)$ \\
\hline $\mathrm{Sb}-\mathrm{O} 1 \mathrm{C}$ & $2,001(5)$ & $\mathrm{O} 1 \mathrm{~B}-\mathrm{Sb}-\mathrm{O} 1 \mathrm{~A}$ & $81,6(2)$ \\
\hline $\mathrm{Sb} \cdots \mathrm{O} 2 \mathrm{C}$ & $3,332(6)$ & $\mathrm{O} 1 \mathrm{~B}-\mathrm{Sb}-\mathrm{O} 2 \mathrm{~A}$ & $79,6(2)$ \\
\hline $\mathrm{Sb}-\mathrm{O} 1 \mathrm{D}$ & $1,987(5)$ & $\mathrm{O} 1 \mathrm{~B}-\mathrm{Sb}-\mathrm{O} 1 \mathrm{D}$ & $87,6(2)$ \\
\hline $\mathrm{Sb} \cdots \mathrm{O} 2 \mathrm{D}$ & $2,687(6)$ & $\mathrm{O} 1 \mathrm{~B}-\mathrm{Sb} \cdots \mathrm{O} 2 \mathrm{D}$ & $118,8(2)$ \\
\hline $\mathrm{Sb}-\mathrm{C} 3$ & $2,096(7)$ & $\mathrm{O} 1 \mathrm{~B}-\mathrm{Sb}-\mathrm{C} 3$ & $98,3(2)$ \\
\hline $\mathrm{C} 1 \mathrm{~A}-\mathrm{O} 1 \mathrm{~A}$ & $1,274(9)$ & $\mathrm{O} 1 \mathrm{C}-\mathrm{Sb}-\mathrm{O} 1 \mathrm{~A}$ & $84,1(2)$ \\
\hline $\mathrm{C} 1 \mathrm{~A}-\mathrm{O} 2 \mathrm{~A}$ & $1,274(9)$ & $\mathrm{O} 1 \mathrm{C}-\mathrm{Sb}-\mathrm{O} 2 \mathrm{~A}$ & $84,3(2)$ \\
\hline $\mathrm{C} 1 \mathrm{~B}-\mathrm{O} 1 \mathrm{~B}$ & $1,320(9)$ & $\mathrm{O} 1 \mathrm{C}-\mathrm{Sb}-\mathrm{O} 1 \mathrm{D}$ & $97,6(2)$ \\
\hline $\mathrm{C} 1 \mathrm{~B}-\mathrm{O} 2 \mathrm{~B}$ & $1,219(10)$ & $\mathrm{O} 1 \mathrm{C}-\mathrm{Sb} \cdots \mathrm{O} 2 \mathrm{D}$ & $77,0(2)$ \\
\hline $\mathrm{C} 1 \mathrm{C}-\mathrm{O} 1 \mathrm{C}$ & $1,340(10)$ & $\mathrm{O} 1 \mathrm{C}-\mathrm{Sb}-\mathrm{C} 3$ & $91,4(2)$ \\
\hline $\mathrm{C} 1 \mathrm{C}-\mathrm{O} 2 \mathrm{C}$ & $1,189(11)$ & $\mathrm{C} 3-\mathrm{Sb} \cdots \mathrm{O} 2 \mathrm{D}$ & $81,5(2)$ \\
\hline C1D-O1D & $1,324(9)$ & $\mathrm{C} 3-\mathrm{Sb} \cdots \mathrm{O} 2 \mathrm{~B}$ & $75,3(2)$ \\
\hline $\mathrm{C} 1 \mathrm{D}-\mathrm{O} 2 \mathrm{D}$ & $1,209(10)$ & & \\
\hline
\end{tabular}


der zweiten Koordinationssphäre zurechnen, wie auch $\mathrm{O} 2 \mathrm{~B}$ und $\mathrm{O} 2 \mathrm{C}$, die noch weiter entfernt sind.

Es überrascht, daß trotz der Vielzahl denkbarer Möglichkeiten keine koordinativen intermolekularen $\mathrm{Sb}-\mathrm{O}$-Kontakte bestehen wie in anderen Organoantimonacetaten [8-10]. Der hier erkennbare Koordinationsunterschied der Acetatgruppen gibt Anlaß, nach Übergangsformen zu Verbindungen ionischen Typs $\left[\mathrm{SbR}_{4}\right]^{+} \mathrm{OOCR}^{-} \mathrm{zu}$ suchen.

\section{Experimenteller Teil}

Die Synthese erfolgte in trockener $\mathrm{N}_{2}$-Atmosphäre. Das ${ }^{1}$ H-NMR-Spektrum wurde bei R.T. und bei $-60{ }^{\circ} \mathrm{C}$ vermessen (Varian T 60). Der Schmelzpunkt wurde thermoanalytisch (Thermal Analyzer Mod. 990, Du Pont) sowie im Kupferblock ermittelt, die $\mathrm{C}, \mathrm{H}$-Werte durch Verbrennungsanalyse und der Sb-Wert atomabsorptionsspektrometrisch bestimmt. Das IR-Spektrum wurde mit einem Perkin-Elmer 283 registriert.

\section{Arbeitsvorschrift}

$2,2 \mathrm{~g}(8,16 \mathrm{mmol})$ Phenyldichlorstiban werden in $30 \mathrm{ml}$ absolutem Methylenchlorid gelöst und dazu $0,66 \mathrm{ml}(8,16 \mathrm{mmol})$ frisch destilliertes Sulfurylchlorid bei $-70{ }^{\circ} \mathrm{C}$ getropft. Man läßt 30 min rühren. Anschließend wird die Lösung spatelweise mit 5,45 g $(32,62 \mathrm{mmol})$ Silberacetat versetzt. Nach einiger Zeit bildet sich ein weißer Niederschlag. Die Reaktionsmischung wird zwei Tage gerührt, dann der Niederschlag über etwas Celite abgefrittet und vom Filtrat das Lösungsmittel abgezogen. Der Rückstand wird in $c a .10 \mathrm{ml}$ Methylenchlorid gelöst, mit Pentan bis zur Trübung versetzt und mehrere Tage im Tiefkühlschrank aufbewahrt.

Die ausgefallen farblosen Kristalle werden abgefrittet und getrocknet.

Ausbeute 59\%; Schmp. $117^{\circ} \mathrm{C}$; C 38,16 (ber. 38,65); H 3,95 (3,93); Sb 28,16 $(27,98) \%$; ${ }^{1} \mathrm{H}-\mathrm{NMR}$ $\left(\mathrm{CH}_{2} \mathrm{Cl}_{2}\right): \delta=2,00 \mathrm{ppm}\left(\mathrm{C}-\mathrm{CH}_{3}, \mathrm{~s}\right) ; \delta=$ $7,38-7,75 \mathrm{ppm}$ (metalpara-H $\left.\mathrm{C}_{6} \mathrm{H}_{5}-\mathrm{Sb}, \mathrm{m}\right) ; \delta=$
7,83-8,22 ppm (ortho- $\mathrm{H} \mathrm{C}_{6} \mathrm{H}_{5}-\mathrm{Sb}, \mathrm{m}$ ); IR $\left(\mathrm{CCl}_{4}\right)$ : 3064 (w); 2927 (m); 2852 (w); 1717 (vst); 1562 (m); $1483(\mathrm{~m}) ; 1439(\mathrm{~s}) ; 1365(\mathrm{~m}) ; 1290(\mathrm{sh}) ; 1250(\mathrm{~s})$; $1008(w) ; 916(\mathrm{w}) ; 726(\mathrm{w}) ; 685(\mathrm{w}) \mathrm{cm}^{-1}$.

\section{Röntgenstrukturanalyse}

Untersucht wurde ein Einkristall mit $0,3 \times 0,4 \times$ $0,15 \mathrm{~mm}^{3}$ (Syntex-P $2_{1}$-Diffraktometer, $\mathrm{MoK}_{\alpha}$-Strahlung, $\lambda=0,71069 \AA$, Graphit-Monochromator). Drehaufnahmen um die kristallographischen Achsen bestätigten die monokline Zellsymmetrie. Eine leastsquares-Rechnung mit 21 zentrierten Reflexen ergab eine Zelle mit $a=8,172(3), b=15,429(7), c=$ $13,902(4) \AA, \beta=92,71(3)^{\circ}, V=1750,9 \AA^{3}, Z=4$ $\left(\varrho_{\text {calc }}=1,65 \mathrm{mg} / \mathrm{mm}^{3}\right)$. Vermessen wurde ein Quadrant der Reflexionssphäre $\left(2 \theta=4-46^{\circ}, \omega\right.$-scan, $\Delta \omega=1,0^{\circ}, 2723$ gemessen, 2409 symmetrieunabhängig, 2001 beobachtet $\left(\mathrm{F}_{\mathrm{o}} \geq 3,92 \sigma\left(\mathrm{F}_{\mathrm{o}}\right)\right)$, Raumgruppe $\mathrm{P} 21 / n$ (Nr. 14); Absorptionskorrektur an Hand von $8 \psi$-scans, Korrekturfaktor von $0,77-1,0)$.

Die Lösung der Struktur erfolgte über PattersonFourier- und Differenzfouriersynthesen. Das Modell wurde über mehrere least-squares-Zyklen verfeinert (208 Variable) bis zu einem $R$-Wert von $R_{1}=0,039$ $(0,051), R_{2}=0,039(0,040)$ mit 2001 (2409) Reflexen (Gewichtung mit $1 / \sigma^{2}$ ). Wasserstoffatome blieben unberücksichtigt. Die höchsten Maxima der Differenzelektronendichte lagen bei $0,5 \mathrm{e} / \AA^{3}$. Sämtliche Rechnungen wurden erstellt mit teilweise modifizierten Programmen des Systems Syntex-XTL unter Verwendung analytisch approximierter Formfaktoren für ungeladene Atome [11] und Berücksichtigung anomaler Dispersion.

Weitere Einzelheiten zur Kristallstrukturuntersuchung können beim Fachinformationszentrum Energie, Physik, Mathematik GmbH, D-7514 Eggenstein-Leopoldshafen 2, unter Angabe der Hinterlegungsnummer 52560, des Autors und des Zeitschriftenzitates angefordert werden.

Der Deutschen Forschungsgemeinschaft und dem Fonds der Chemischen Industrie danken wir für die finanzielle Unterstützung der Arbeit.

[6] H. A. Meinema und J. G. Noltes, J. Organomet. Chem. 36, 313 (1972).

[7] D. B. Sowerby, M. J. Begley und P. L. Millington, J. Chem. Soc. Chem. Commun. 478, 896 (1984).

[8] M. Wieber, D. Wirth und Ch. Burschka, Z. Naturforsch. 39b, 600 (1984).

[9] S. B. Bone und D. W. Sowerby, J. Organomet. Chem. 184, 181 (1980).

[10] M. Hall und D. B. Sowerby, J. Chem. Soc. 1980, 1292.

[11] D. T. Cromer und J. T. Waber, Int. Tables for X-rayCrystallography, Vol. IV, pp. 99-101, Kynoch Press, Birmingham (1974). 